\title{
Mucocutaneuse (Palpebral and Gingival) Primary Amyloidosis: A Case Report
}

\author{
Abdolali Shahrasbi*, Yasaman Vakiloroaya and Yasamin Rekabdar \\ Hematology/Oncology department, Bouali hospital, Islamic Azad University, Iran
}

Received: April 23, 2018; Published: May 04, 2018

*Corresponding author: Abdolali Shahrasbi, Hematology/Oncology department, Bouali hospital, Tehran Medical Sciences Branch, Islamic Azad University, Tehran Iran

\section{Abstract}

Introduction: Amyloidosis is a rare disease resulting from extracellular accumulation of abnormal insoluble protein fibrils. AmylUidosis classified into two types primary(AL) and secondary(AA). Primary amyloidosis(AL) is a multiple myeloma associated disease. The primary localized cutaneus amyloidosis (PLCA) is a kind of of primary amyloidosis with isolated amyloid deposition in the skin and mucous membrane. Case discussion: We described a case of PLCA with a long history of palpebral and gingival involvement. There are a few treatment modalities for these groups of patients such as local therapy or systemic medical therapy. Conclusion: Although our patient has recieved multiple lines of immunosuppressive treatment during long period of time, the disease progressed continously and disturbed her visual field.

\section{Introduction}

Amyloidosis is a rare disease resulting from extracellular accumulation of abnormal insoluble protein fibrils called amyloid which distinguish by apple green birefringence on congo red histological staining [1]. Amyloidosis classified into primary(AL) or secondary(AA) types which can occurs in multi organs (systemic amyloidosis) or limited to one organ (localized amyloidosis). AA amyloidosis occurs in various chronic inflammatory disorders while AL type is multiple myeloma-associated [2]. The primary localized cutaneus amyloidosis (PLCA) is a condition of primary amyloidosis with isolated amyloid deposition in the skin. PLCA can appear in the forms of macular, papular and rarely nodular. Gingival is also a very rare site of localized amyloidosis in head and neck area and inconsequentially in oral cavity [3]. In this case report we present a case of primary amyloidosis with involment of both eyelids and gingiva.

\section{Case presentation}

A 48-year old female came to our clinic 10 years ago with bilateral palpebral and gingival evolving mucosal lesions that they had developed from 1-2 month before. The pathology report of eyelid skin and gingival buccal mucosa revealed deposition of fragmented amorphous materials in the dermis and gingival mocusa that exhibit an apple green birefringence in viewing of the Congo red staining. There was no history of preceding trauma or any specific disease in her history. Physical examination showed popular erythema to shiny brown color non-itching palpebral skin and patchy lesions of gingiva. There was no pain, and palpebral sensation and movement were intact. Laboratory tests including serum protein electrophoresis and urine protein electrophoresis showed Gamma peak without light chain proteinuria and other biochemical tests such as $\mathrm{CBC}, \mathrm{Ca}, \mathrm{BUN}$ and Creatinine were normal. ESR was elevated.

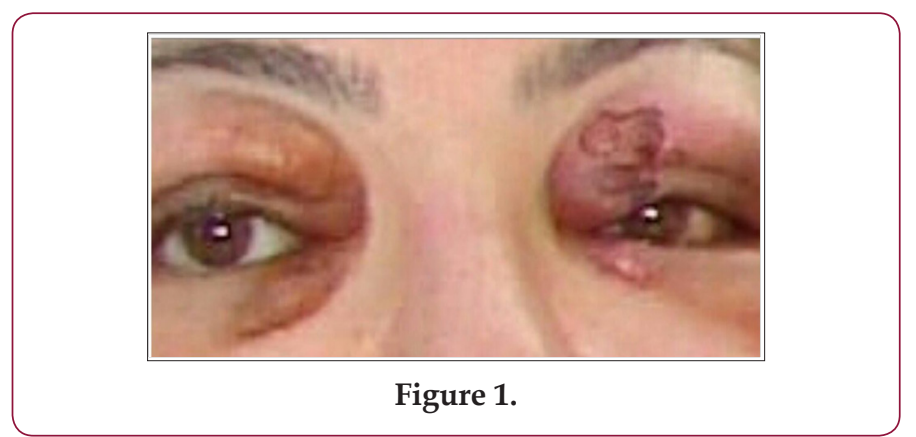

We also did an extensive study in order to rule out systemic amyloidosis. Bone marrow biopsy revealed around 10\% plasma cytosis, abdomen-pelvic CT Scan, CXR, were normal and skull x-ray didn't show any evidence of lytic lesions. All the laboratory data and final histological findings and periodic reevaluation confirm that the disease localized to palpebral and gingival regions (Figure 1). Then we decided to prescribe alkeran and prednisolone as main trance therapy with the lowest dose and replaced thalidomide instead of alkeran. Unfortunately, the patient didn't keep on following up regularly and eyelid lesions reappeared again, and we prescribed VTD regimen (Velcade, Thalidomide, Dexamethazon) after one year and a half. VTD continued for 12 weeks, although it was well 
tolerated response was partial. Eyelid lesions were persistent but decreased in size and erythemato color (Figure 2).

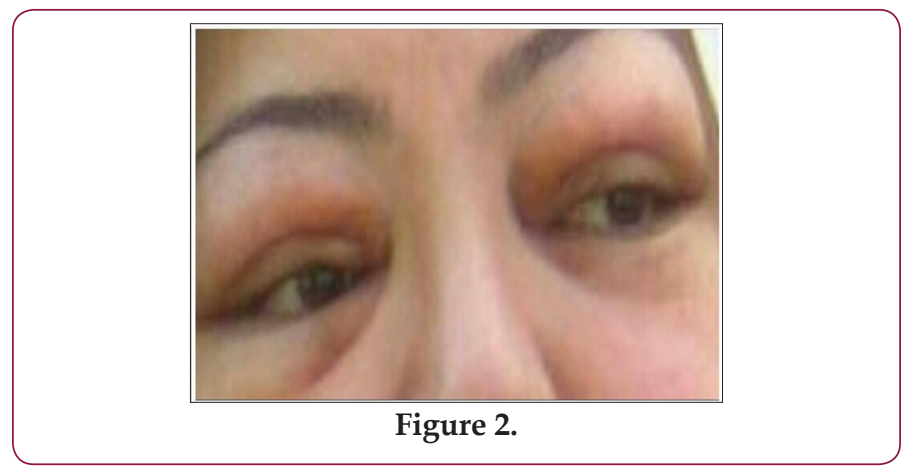

Treatment cycles continued for next 3 months and then she was followed just by thalidomide and aspirin. With the passing of the years (10 years) she had received on/off periodic treatment with thalidomide, (lenalidomide), dexamethasone and aspirin and we also referred her to a reconstructive surgeon. The operation was not done due to possible complications of operation and worries about the healing process and scar tissue. After ten years she has been presenting with enlarged and deeply erythemato popular lesions in both eyelids which interfering with her visual field (Figure 3).

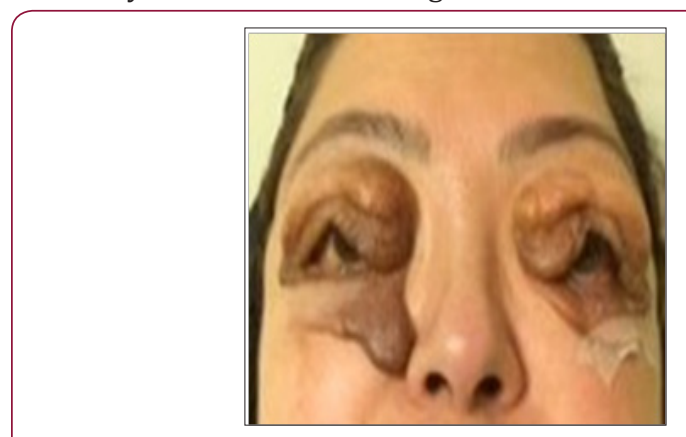

Figure 3.

\section{Discussion}

Primary palpebral amyloidosis is a rare condition. It maybe misdiagnosed due to its clinical manifestations which leads us to different diagnosis include basal cell carcinoma, squamouse cell carcinoma, lacrimal gland carcinoma or lymphoma [4] Histological examinations and immunohistochemistry tests with immunoglobulin light chain and cytokeratin antibodies are the first diagnostic approach to correct diagnosis of primary localized cutaneuse amyloidosis. Reports showed that there is 5-50\% risk of progression to systemic disease for PLCA. Thus, rule out systemic progression should be done by frequent monitoring including the complete history, physical examinations, serume protein electrophoresis, urine protein electrophoresis, imaging evaluation and other laboratory tests such as CBC, BUN, crearinin, Ca, ESR. There are multiple treatment plans for PLCA such as surgery, multiple immunosuppressive agents, intralesional injection methotrexate, carbon dioxide laser and cryotherapy $[5,6]$.

Reports show that carbon dioxide laser and cryotherapy are not useful due to persistent of amyloid deposition. Although there aren't enough experiences intralesional methotrexate injection may have some benefits. Surgical operation is not recommended for management of PLCA because there is a risk of recurrence in any localized excision and poor cosmetic results and increased ocular complaints. Surgical procedure was not our preferred treatment [7].

\section{Conclusion}

We have used multiple lines of standard immunosuppressive therapy for our patient to limit eyelid lesions but it seems that in spite of passing 10 years and on/off treatment courses natural history of the disease has been continuously progressive with partial response rate.

\section{References}

1. Al Raddadi R, Abbaspour V, Ndiaye M (2016) Papular Cutaneous Amyloidosis Associated with Multiple Myeloma. Science Journal of Clinical Medicine 5(2): 16-19.

2. Bucci T, Bucci E, Rullan AMP, Bucci P, Nuzzolo P (2014) Localized amyloidosis of the upper gingiva: a case report. Journal of Medical Case Reports 8: 198.

3. Kumar S, Sengupta RS, Kakkar N, Sharma A, Singh S, et al. (2013) sSkin Involvement in Primary Systemic Amyloidosis. Mediterranean Journal of Hematology and Infectious Diseases 5(1): e2013005.

4. Caggiati A, Campanella A, Tenna S, Cogliandro A, Potenza A, et al. (2010) Primary Amyloidosis of the Eyelid: A Case Report. In Vivo 24(4): 575578.

5. Haverkampf S, Evert K, Schröder J, Schreml S (2016) Nodular Cutaneous Amyloidosis Resembling a Giant Tumor. Case Reports in Dermatology $8(1): 22-25$.

6. Raymond J, Choi J (2016) Nodular cutaneous amyloidosis effectively treated with intralesional methotrexate. JAAD Case Reports 2(5): 373376.

7. Oliveira Ederson Vale Lopes de, Poszetti Ana Carolina Garcia, Pozetti Rubrics Maria de Oliveira, Antonio Joao Roberto, Michalany Nilceo Schwery (2012) Primary systemic amyloidosis associated with multiple myeloma. An Bras Dermatol 87: 119-122.

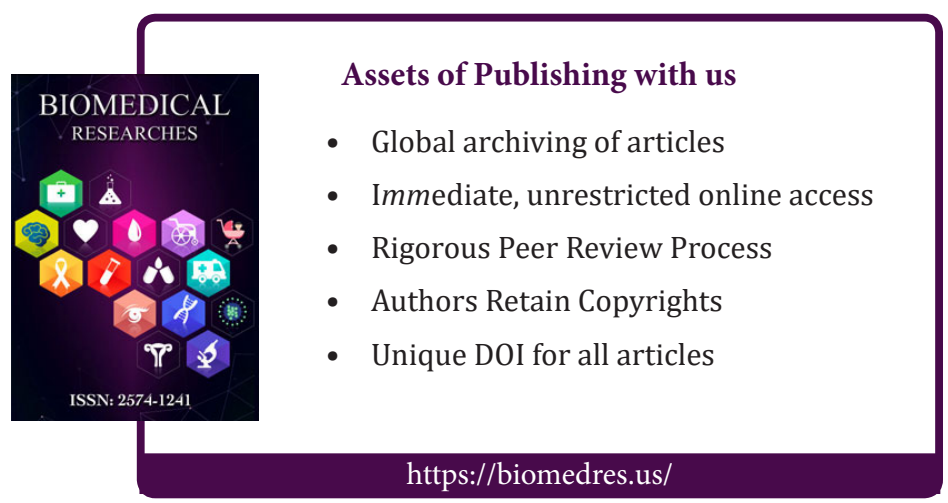

